\title{
Globally Stable Microresonator Turing Pattern Formation for Coherent High-Power THz Radiation On-Chip
}

\author{
Shu-Wei Huang, ${ }^{1, *}$ Jinghui Yang, ${ }^{1, \dagger}$ Shang-Hua Yang, ${ }^{2}$ Mingbin Yu, ${ }^{3}$ Dim-Lee Kwong, ${ }^{3}$ T. Zelevinsky, ${ }^{4}$ \\ Mona Jarrahi, ${ }^{2}$ and Chee Wei Wong ${ }^{1, *}$ \\ ${ }^{1}$ Fang Lu Mesoscopic Optics and Quantum Electronics Laboratory, University of California, \\ Los Angeles, California 90095, USA \\ ${ }^{2}$ Terahertz Electronics Laboratory, University of California, Los Angeles, California 90095, USA \\ ${ }^{3}$ Institute of Microelectronics, A*STAR, Singapore 117865, Singapore \\ ${ }^{4}$ Department of Physics, Columbia University, New York, New York 10027, USA \\ (Received 9 August 2016; revised manuscript received 24 May 2017; published 5 October 2017)
}

\begin{abstract}
In nonlinear microresonators driven by continuous-wave (cw) lasers, Turing patterns have been studied in the formalism of the Lugiato-Lefever equation with emphasis on their high coherence and exceptional robustness against perturbations. Destabilization of Turing patterns and the transition to spatiotemporal chaos, however, limit the available energy carried in the Turing rolls and prevent further harvest of their high coherence and robustness to noise. Here, we report a novel scheme to circumvent such destabilization, by incorporating the effect of local mode hybridizations, and we attain globally stable Turing pattern formation in chip-scale nonlinear oscillators with significantly enlarged parameter space, achieving a record-high power-conversion efficiency of $45 \%$ and an elevated peak-to-valley contrast of 100 . The stationary Turing pattern is discretely tunable across $430 \mathrm{GHz}$ on a THz carrier, with a fractional frequency sideband nonuniformity measured at $7.3 \times 10^{-14}$. We demonstrate the simultaneous microwave and optical coherence of the Turing rolls at different evolution stages through ultrafast optical correlation techniques. The free-running Turing-roll coherence, $9 \mathrm{kHz}$ in $200 \mathrm{~ms}$ and $160 \mathrm{kHz}$ in 20 minutes, is transferred onto a plasmonic photomixer for one of the highest-power $\mathrm{THz}$ coherent generations at room temperature, with $1.1 \%$ optical-to- $\mathrm{THz}$ power conversion. Its long-term stability can be further improved by more than 2 orders of magnitude, reaching an Allan deviation of $6 \times 10^{-10}$ at $100 \mathrm{~s}$, with a simple computer-aided slow feedback control. The demonstrated on-chip coherent high-power Turing-THz system is promising to find applications in astrophysics, medical imaging, and wireless communications.
\end{abstract}

DOI: 10.1103/PhysRevX.7.041002

The spontaneous formation of stationary periodic patterns from homogeneous backgrounds first elucidated by Turing has served as the basis for developmental biology morphogenesis, chemical kinetics far from equilibrium, and the formation of fractals and chaos in nonlinear dynamics [1-3]. Of both conceptual importance and practical interest, optical Turing pattern formation has been theoretically proposed and investigated in continuous-wave (cw)-laser-pumped Kerr-active microresonators [4-7], with emphasis on its high coherence and exceptional robustness against perturbations. Generally, the Kerr-active microresonator is designed to possess anomalous group velocity dispersion (GVD) for convenient phase-matching fulfillment, and the

\footnotetext{
*swhuang@seas.ucla.edu

†yangjh@seas.ucla.edu

¥cheewei.wong@ucla.edu
}

Published by the American Physical Society under the terms of the Creative Commons Attribution 3.0 License. Further distribution of this work must maintain attribution to the author(s) and the published article's title, journal citation, and DOI.
Subject Areas: Nanophysics, Nonlinear Dynamics, Optics

formation dynamics of the spontaneous patterns can be described by the Lugiato-Lefever equation [4]. As the driving laser is frequency tuned into the cavity resonance from the blue side, stable Turing rolls first spontaneously emerge from the background, then quickly destabilize into spatiotemporal chaos [8,9], and eventually transition into dissipative Kerr solitons [10-14] or Kerr frequency combs [15-18] (Fig. S1 in Ref. [19]). We note that the Turing roll in this dispersion regime, despite its optimally coherent properties [20], only exists in a limited phase space, and its quick destabilization into chaos limits the attainable powerconversion efficiency [Figs. 1(a) and 1(b)], preventing further harvest of the high coherence and the noise robustness of the Turing pattern.

To expand the stability zone and attain higher power conversion, we approach the spontaneous Turing pattern formation in a distinctly different way. Our Kerr-active microresonator is designed to possess a large globally normal GVD of $100 \mathrm{fs}^{2} / \mathrm{mm}$ (Fig. S2 in Ref. [19]); thus, phase matching is strictly forbidden unless local dispersion anomalies are introduced to the system, providing additional GVD to balance the nonlinearity locally. In our high 

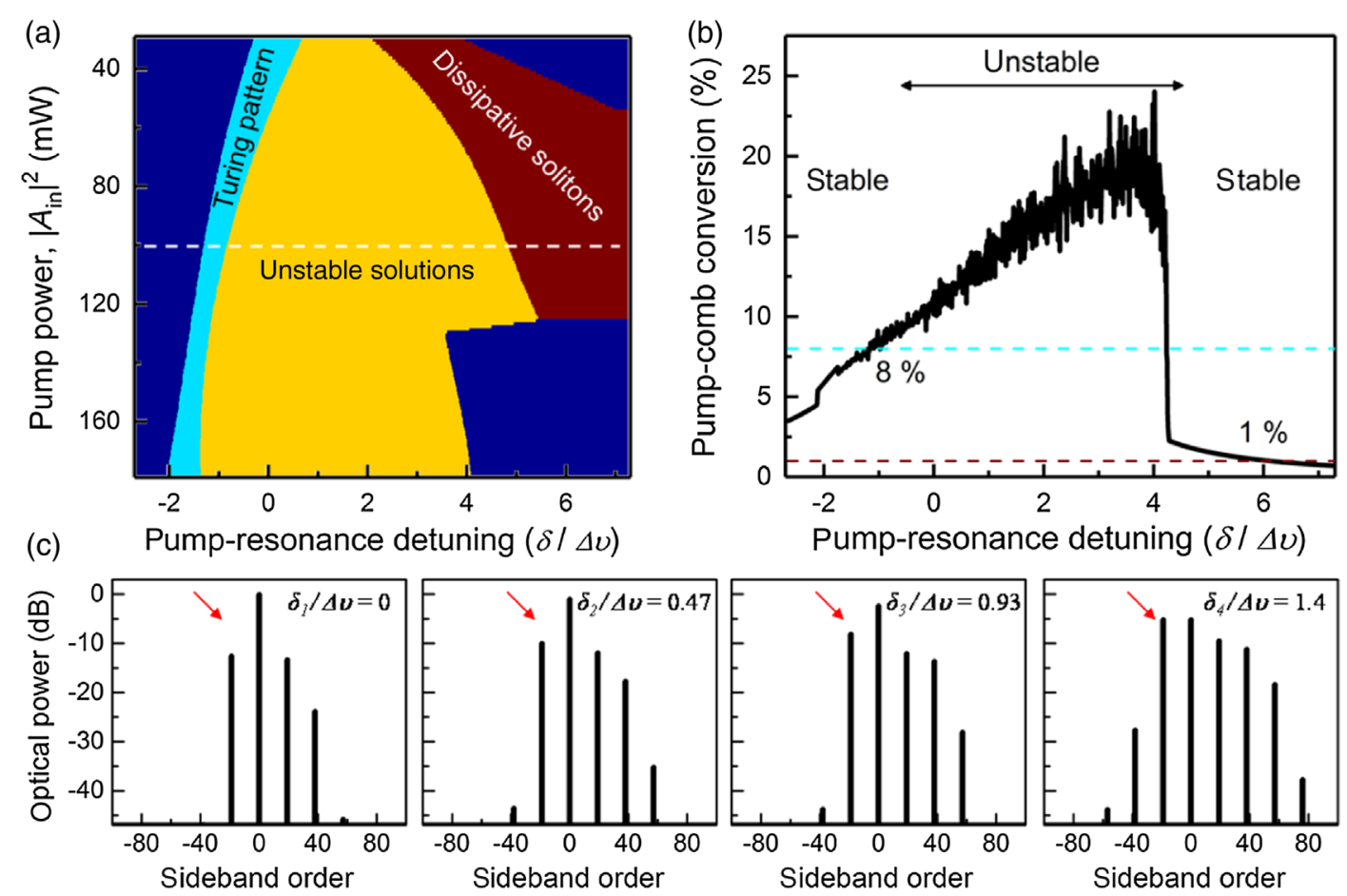

FIG. 1. (a) Stability diagram of the Turing pattern in the anomalous GVD regime. Light blue shows the region of the stable Turing pattern, yellow shows the region of breathers and spatiotemporal chaos, and red shows the region of the soliton and soliton molecules. (b) Pump-to-comb power-conversion efficiency along the white dashed line in (a), showing only $8 \%$ maximum power conversions to the Turing pattern before destabilization and transition to chaos happens. (c) Simulation of the Turing roll in the normal dispersion microresonator $\left(1300 \mathrm{fs}^{2}\right.$ ), where the Turing roll is excited by local mode hybridization. The red arrow points to the mode where local dispersion disruption is introduced in the Lugiato-Lefever model. It shows an apparent asymmetry and can be tuned further into resonance without triggering the subcomb growth and the associated Turing pattern destabilization, expanding the stability zone and opening the route to harness Turing pattern's high coherence and exceptional robustness at high optical powers. Note that the pumpresonance detuning used in the simulation is referenced to the cold cavity resonance frequency.

$Q$ microresonator (loaded quality factor of $3.7 \times 10^{5}$ ), such anomalies result from the hybridization of two transverse modes $\left(\mathrm{TM}_{11}\right.$ and $\left.\mathrm{TM}_{21}\right)$ with distinct free spectral ranges (FSRs) when their resonant frequencies are in the vicinity of each other [21-27]. The spectral position of the mode hybridization defines the Turing pattern formation dynamics, and it can be changed by the design of the FSR difference. As the balance between the GVD and the Kerr nonlinearity is only fulfilled locally in the confined spectral range where mode hybridization occurs, subcomb growth and subsequent destabilization of the Turing pattern is avoided [Fig. 1(c)]. By enabling the deeper driving into resonance without transition into chaos or soliton states, the conversion efficiency from pump to Turing pattern can thus be significantly enhanced in our system. The mode-hybridization-mediated phase matching - by adjusting the relative frequency between the pump mode and the mode hybridization positionfurther enables the repetition rate of the Turing roll to be discretely tunable. Moreover, the Turing-roll spectra can exhibit controllable asymmetry through registering the pump mode on different sides of the first local-mode hybridization region $\varepsilon_{1}$ [Fig. 2(a)].
Here, we demonstrate, for the first time, the scheme of incorporating the mode hybridization effect to attain globally stable microresonator Turing patterns in chip-scale nonlinear nitride cavities with significantly enlarged parameter space, achieving an unprecedented pump depletion and a record-high power-conversion efficiency of $45 \%$ with an elevated peak-to-valley contrast of 100 . We interrogate the commensurate and coherent nature of the spontaneous dissipative structure with ultrafast optical intensity autocorrelation, microwave spectral noise analysis, and heterodyne beating against a benchmark fiber frequency comb. The fractional-frequency-sideband non-uniformity of the Turing pattern is measured at $7.3 \times 10^{-14}$, with a short-term (200 ms sweep time) linewidth of $9 \mathrm{kHz}$ and a long-term (over 20 minutes) fluctuation of $160 \mathrm{kHz}$ in the free-running mode. The long-term stability can be further improved by more than 2 orders of magnitude, reaching an Allan deviation of $6 \times 10^{-10}$ at $100 \mathrm{~s}$, with a simple computeraided slow feedback control. Towards THz applications, we then transfer the Turing pattern optical coherence to the $\mathrm{THz}$ carrier through a plasmonic ErAs:InGaAs photomixer, generating up to $600 \mu \mathrm{W} \mathrm{THz}$ radiation power at room temperature. The carrier frequency is discretely tunable over 

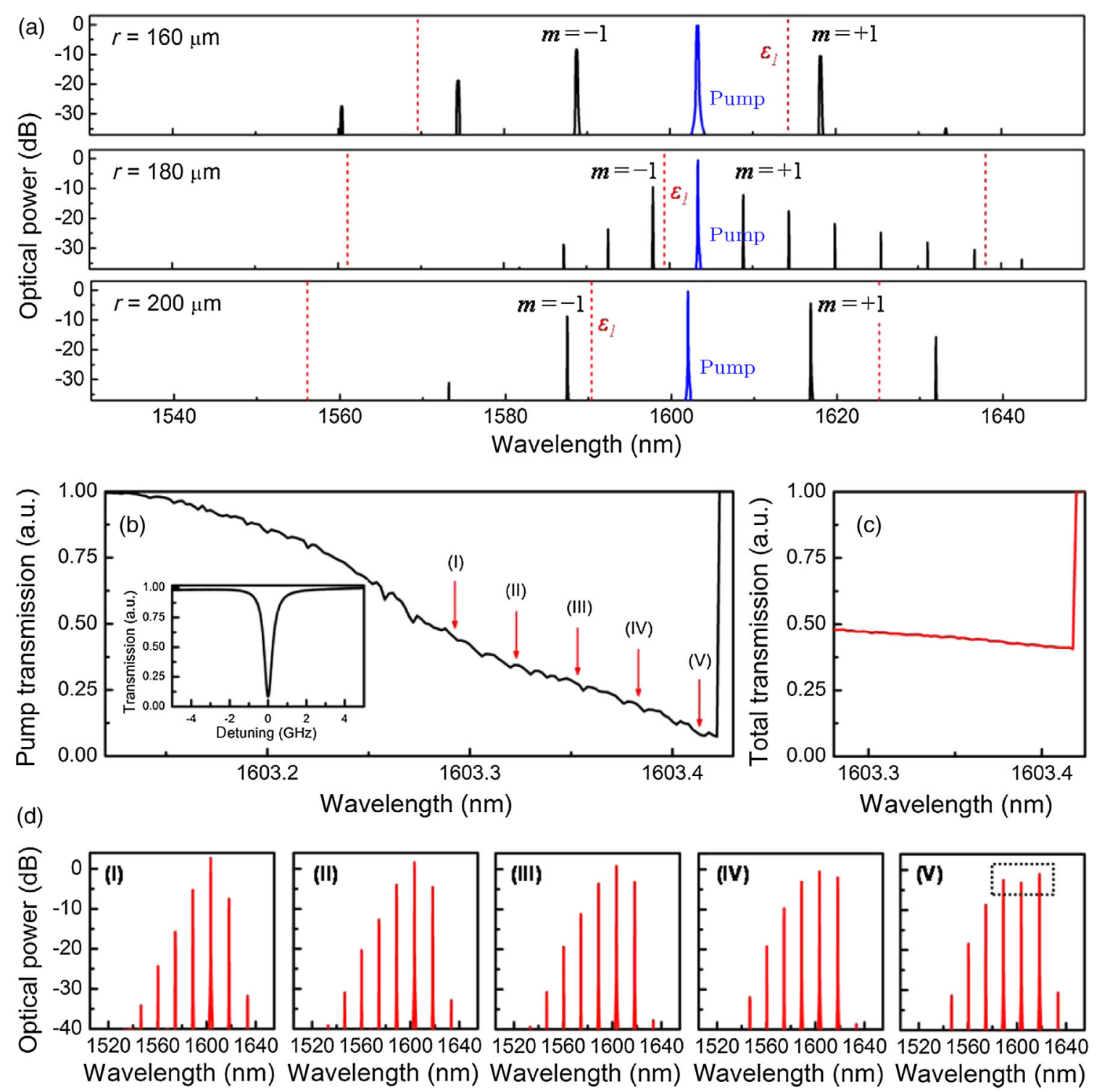

FIG. 2. (a) Turing rolls generated from ring resonators with different radii. Even though the GVD of the ring resonators differ by less than $2 \mathrm{fs}^{2} / \mathrm{mm}$, the $\mathrm{TM}_{11}-\mathrm{TM}_{21}$ mode hybridization positions (red dashed lines) with respect to the pump (blue lines) shift because of the change in the ring radii, resulting in abrupt dispersion variations locally and very different spontaneous Turing patterns. The Turingroll repetition rates are $1.72 \mathrm{THz}(12 \times \mathrm{FSR})$ for the $160 \mu \mathrm{m}$ radius ring, $0.64 \mathrm{THz}(5 \times \mathrm{FSR})$ for the $180 \mu \mathrm{m}$ radius ring, and $1.72 \mathrm{THz}$ $(15 \times$ FSR) for the $200 \mu \mathrm{m}$ radius ring. (b) Pump-cavity transmission as a function of the pump wavelength, labeling the detunings where different Turing-roll stages are generated. Inset: The cold resonance of the pump mode, measuring a loaded Lorentzian linewidth of $500 \mathrm{MHz}$ and a loaded quality factor of $3.7 \times 10^{5}$. (c) Total cavity transmission as a function of the pump wavelength in the range where the Turing roll is generated. Compared to the pump-cavity transmission, the total cavity transmission shows a less apparent decrease as the pump is tuned into the resonance, confirming an efficient power conversion from the pump to the generated Turing lines. (d) Example Turing-roll spectra at different stages. At stage V, even a highly depleted pump close to the resonance is observed in the measurement, illustrated in the dashed box.

a broadband from $1.14 \mathrm{THz}$ to $1.57 \mathrm{THz}$. The demonstrated coherent high-power Turing-THz system offers the potential to be the room-temperature on-chip $\mathrm{THz}$ local oscillator for astrophysics, medical imaging, and wireless communication [28-33].

Here, a TM-polarized cw laser with an optical power of $29.5 \mathrm{dBm}$ is frequency tuned from the blue side of the cavity resonance to trigger the Turing pattern formation. The measured spectra of the spontaneous Turing patterns generated from ring resonators with different radii are shown in Fig. 2(a), with the pump illustrated in blue and the mode hybridization positions labeled as red dashed lines. Despite very similar GVD $\left(\beta_{2}\right)$ from different radii ring resonators, the Turing patterns show spectral shapes that are distinct from each other. Specifically, the spectral lines on the side of the first mode hybridization position are suppressed because 
of the increasing phase mismatch associated with the modehybridization-induced local dispersion disruption. With the first sideband pair $(m= \pm 1)$ phase matched because of the additional contribution from the mode hybridization on mode 1 , the phase-matching condition can be written as

$$
\Delta k\left(2 \omega_{0}-\omega_{1}-\omega_{-1}\right)=\beta_{2} \Delta^{2}+\gamma P_{\text {int }}-\varepsilon=0,
$$

where $\varepsilon$ represents the contribution from the mode hybridization, $\Delta$ the Turing-roll repetition rate, $\gamma$ the nonlinear Kerr coefficient, and $P_{\text {int }}$ the intracavity power. Here, $k_{1}=k_{0}+$ $\beta^{\prime} \Delta+\left(\beta_{2} / 2\right) \Delta^{2}+\varepsilon$ and $k_{m}=k_{0}+\beta^{\prime}(m \Delta)+\left(\beta_{2} / 2\right)(m \Delta)^{2}$, where $\beta^{\prime}$ is the group velocity. Then, the phase-matching condition for the first cascaded four-wave mixing on either side of the pump can be written as

$$
\begin{aligned}
\Delta k\left(2 \omega_{1}-\omega_{2}-\omega_{0}\right) & =\beta_{2} \Delta^{2}+\gamma P_{\text {int }}+2 \varepsilon=3 \varepsilon, \\
\Delta k\left(2 \omega_{-1}-\omega_{0}-\omega_{-2}\right) & =\beta_{2} \Delta^{2}+\gamma P_{\text {int }}=\varepsilon .
\end{aligned}
$$

The phase mismatch on the side of the mode-crossing position is 3 times larger than the other process; thus, the symmetry of the Turing-roll spectra is broken. Similar symmetry breaking by mode hybridization has also been demonstrated in microwave photonics recently [34]. Turing-roll repetition rates also show dramatic variations $(\approx 640 \mathrm{GHz}$ in the $180 \mu \mathrm{m}$ radius ring and $\approx 1.72 \mathrm{THz}$ in the $160 \mu \mathrm{m}$ and $200 \mu \mathrm{m}$ rings) that cannot be solely explained by the change in the cavity round-trip time. These features are direct consequences of the unique phase-matching configuration employed in our design, with critical roles in the efficient coherence transfer from Turing pattern to $\mathrm{THz}$ radiation to be detailed later. Note that the modehybridization-induced local dispersion disruption $\varepsilon$ can be dynamically tuned by changing the temperature of the microresonator, thereby providing an additional dimension to control the Turing pattern formation dynamics (detailed in Ref. [19]).

Next, we focus on the results generated from the $160 \mu \mathrm{m}$ radius ring because of its energy concentration in the wavelength range shorter than $1570 \mathrm{~nm}$, overlapping better with the spectral response of our plasmonic photomixer discussed later. Figures 2(b) and 2(c) show the pump and total transmitted intensities, measured simultaneously for different detunings. The pump transmission shows a triangular tuning curve with a strong dip from normalized unity into $\approx 10 \%$ of the original transmission, while the total transmission shows only a small drop from $48 \%$ to $41 \%$ this provides evidence of the efficient total energy transfer from the pump into the complete Turing pattern sidebands. To examine this further, Fig. 2(d) plots the corresponding Turing-roll spectra at different detuning stages: Stable spontaneous Turing patterns are observed without any sign of destabilization at all detunings. The power-conversion efficiency, defined as the integrated power of the output Turing roll divided by the on-chip pump power, reaches as high as $45 \%$ at stage III (detailed in Ref. [19]). Closer to resonance at stage $\mathrm{V}$, an even stronger pump depletion is achieved, with the pump intensity $2 \mathrm{~dB}$ lower than even the first modulation sidebands.

We conduct a series of ultrafast optical intensity autocorrelation (IAC) measurements, as shown in Fig. 3(a), to investigate the temporal structure of the Turing patterns at different evolving stages. At all the stages, stable and strong quasisinusoidal oscillations are observed. While pumping closer to resonance results in a monotonic increase in the pump depletion [Fig. 2(d)], counterintuitively, the IAC traces show a discernible minimum background between stage II and stage III. As elaborated on later, it is a direct consequence of strong pump depletion. At stage III, a peakto-valley intensity contrast ratio of more than 100 is achieved (Fig. S8 in Ref. [19]). We next perform extensive measurements to examine the coherence of the spontaneous Turing formation, illustrated in Figs. 3(b)-3(e). The RFamplitude noise spectra of the Turing pattern up to $3 \mathrm{GHz}$, 6 times the cavity linewidth, shows an absence of RF peaks and a noise level at the instrumentation detection background limit, indicative of the existence of a single Turingroll family with commensurate repetition rates [Fig. 3(b)]. We further heterodyne beat the Turing sidebands $(m= \pm 1$ and pump) against a benchmark fiber frequency comb (see Appendix C) and perform ratio counting of the sidebands to interrogate the frequency uniformity, which sets the fundamental limit on the coherence transfer from the Turing pattern [Fig. 3(c)]. When the Turing-roll repetition rate is made nondivisible by the fiber frequency-comb spacing, the beat frequencies of consecutive sidebands will be an arithmetic sequence. Namely, $\delta_{2}=\delta_{1}+\Delta=\delta_{0}+2 \Delta$. Here, we make the common difference $\Delta$ equal to $1 \mathrm{MHz}$. Ideally, the ratio between $\delta_{2}-\delta_{0}$ and $\delta_{1}-\delta_{0}, R$, should be 2 , and deviation from this ratio, $\varepsilon_{R}$, is a measure of the sideband frequency nonuniformity, $\varepsilon=\varepsilon_{R} \cdot \Delta$. Excellent sideband uniformity of the Turing pattern is observed at all evolving stages, with the average nonuniformity measured at $125 \mathrm{mHz}$, or $7.3 \times 10^{-14}$ when referenced to the Turing pattern repetition rate at $1.72 \mathrm{THz}$ [Fig. 3(c)]. Figure 3(d) next shows the self-heterodyne beat note of the first sideband (see Appendix C), demonstrating the Turing lines down to a pump-coherence-limited linewidth of $500 \mathrm{kHz}$. The linewidth measurements independently confirm the good coherence of the Turing rolls at all detunings. Figure 3(e) also shows the real-time power monitoring of the four strongest sidebands. All sidebands present similar intensity noise of less than $1 \%$ (integrated from $100 \mathrm{~Hz}$ to $100 \mathrm{MHz}$ ), and no cyclic energy exchange between sidebands is observed, excluding the possibility of breathing dynamics with conserved total power and supporting the evidence of stationary Turing pattern formation with an extensive stability zone.

To examine whether the temporal shape of the spontaneous Turing pattern is subjected to the perturbation in the initial condition and the pump detuning scan, we performed 

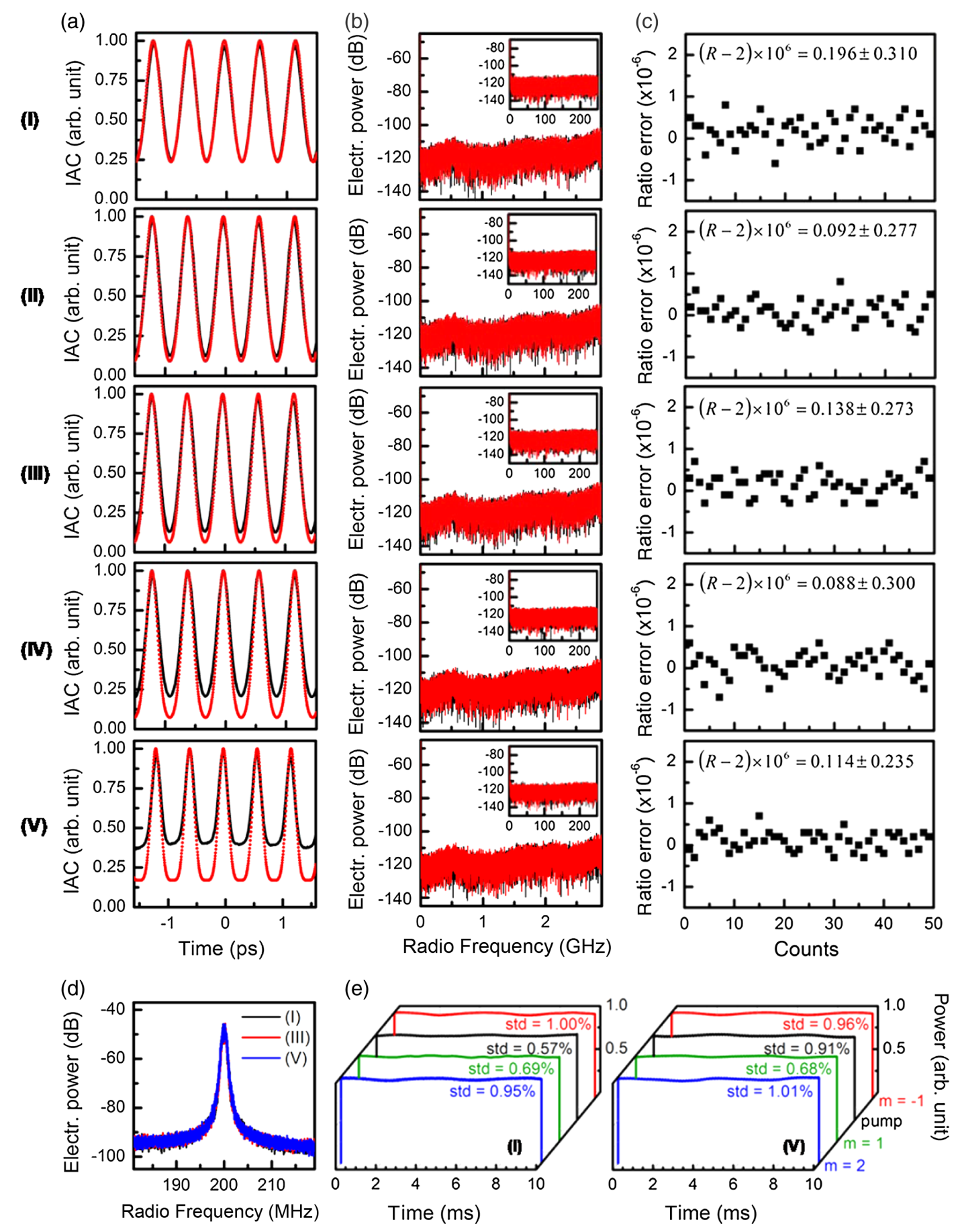

FIG. 3. (a) IAC traces of the Turing rolls at different stages. The red dashed lines are the ideal traces calculated from the spectra, while the black curves are the measured traces. As the sidebands grow, the deviations between the measured and calculated IAC traces also increase. (b) To examine the emergence of incommensurate subcombs, RF amplitude noise spectra of the Turing rolls (black curve) along with the detector background (red curve) are measured up to $3 \mathrm{GHz}, 6$ times the cavity linewidth. No apparent amplitude noise is observed, verifying the existence of the commensurate subcombs. Inset: Zoom-in RF amplitude noise spectra up to $250 \mathrm{MHz}$, at the instrumentation detection noise floor. (c) To probe the equidistance of the Turing rolls, the beat notes between the three Turing-roll sidebands (pump, $m=1$, and $m=2$ ) and the adjacent fiber-laser frequency-comb lines are measured, and the ratio errors are presented. The small deviation from the ideal ratio $R$ of 2 [ $R$ defined in the main text as $\left.\left(\delta_{2}-\delta_{0}\right) /\left(\delta_{1}-\delta_{0}\right)\right]$ verifies the Turing pattern's excellent uniformity. The average nonuniformity is measured at $125 \mathrm{mHz}$, or $7.3 \times 10^{-14}$ when referenced to the Turing pattern repetition rate at $1.72 \mathrm{THz}$. (d) The linewidth of the first sideband is measured at $500 \mathrm{kHz}$, limited by the coherence of the pump laser, by the selfheterodyne technique at different stages. No linewidth broadening is observed, independently confirming that the coherence of the Turing roll is maintained at all evolving stages. (e) Power fluctuation of individual sidebands at the stages I (left) and V (right) with a sampling rate of $250 \mathrm{MHz}$, ruling out the possibility of breathing solutions. 
the IAC measurements at three different tuning speeds and two independent starts. Each of the Turing IAC dynamics remains identical (Fig. S9 in Ref. [19]), illustrating the good robustness of the Turing patterns. To understand the dynamics better, ideal IAC traces from transform-limited Turing patterns are superimposed onto the measured IAC traces [Fig. 3(a), in red]. As the Turing pattern is driven closer to resonance, there is increasing discrepancy of the measured pattern from the transform limit. The change of temporal shapes without the coherence loss implies that the spectral phase of the Turing pattern varies at the different evolving stages; thus, different external-phase-compensation strategies are necessary if the temporal properties of the Turing pattern are to be fully utilized. The spectral phase variation can be understood as the consequence of the pump phase slip around the resonance. The relationship between the output and the intracavity pump power can be written as

$$
A_{p, \text { out }}=-\frac{\gamma_{c}-\gamma_{\alpha}-i \delta}{\sqrt{2 \gamma_{c}}} \sqrt{T_{R}} A_{p, \text { cav }},
$$

where $\gamma_{c}$ and $\gamma_{\alpha}$ are the half-width half-maximum linewidths associated with the coupling losses and the intrinsic cavity losses, respectively. In our microresonator, $\gamma_{c}=160 \mathrm{MHz}$ and $\gamma_{\alpha}=90 \mathrm{MHz}$. The output pump will experience a $\pi$ phase shift as it traverses through the resonance (Fig. S9a in Ref. [19]). Such a phase slip is due to the interference between the intracavity and the input pump; thus, the other sidebands of the Turing roll will not experience such a phase shift. This additional pump phase slip results in the observed change of temporal structure. The distinct responses to dispersion at different Turing states (illustrated in Fig. S10 in Ref. [19]) are well captured by considering the pump phase offset (Fig. S11b in Ref. [19]). Note that most of the phase slip happens very close to cavity resonance; thus, the observation of its effect is attributed to the unique design of our microresonator, which utilizes the local mode hybridization to fulfill the phase matching of spontaneous Turing pattern formation and greatly suppresses the Turing pattern destabilization even when the pump is deep into the resonance.

The robustness, tunability, good coherence, and high efficiency of the demonstrated Turing roll make it an excellent photomixer pump for narrow-linewidth-tunable $\mathrm{THz}$ radiation. Different from deriving the pump from a mode-locked laser [32], our Turing pattern offers the advantage of efficient power use and reduced system complexity as its quasisinusoidal intensity profile (Fig. S8 in Ref. [19]) is directly applicable as a photomixer pump. Other photomixer pump sources [35] include independent lasers with frequency stabilization [36,37], single lasers with active high-speed phase modulation [31], and tunable dual-mode lasers [38-42]. While phase locking two independent lasers to external frequency references can potentially provide the ultimate coherence, it suffers from greatly increased system complexity. Using a single laser with active phase modulation simplifies the system, but high-speed phase modulation with a bandwidth higher than $1 \mathrm{THz}$ is technologically challenging. A tunable dual-mode laser is attractive in its compact footprint and broadband tunability; however, it has a large long-term frequency drift due to the uncommon paths taken by the two modes, and solving it again requires a sophisticated phase-locking technique. Our demonstrated Turing roll provides an alternative platform as it not only is intrinsically compatible with high power operation but also offers a balance between highest coherence and lowest system complexity.

To characterize the stability of the free-running Turingroll repetition rate, which determines the linewidth and fluctuation of the $\mathrm{THz}$ radiation, we beat the pump and one of the sidebands with the adjacent fiber-laser frequencycomb lines and electrically mix the two signals to get the beat note at the frequency difference, as shown in Fig. S13 in Ref. [19]. Figure 4 shows the long-term frequency fluctuation, measuring a root-mean-square frequency fluctuation of $160 \mathrm{kHz}$ over 20 minutes. The left inset shows the linewidth of the beat note with a sweep time of $200 \mathrm{~ms}$, measuring a narrow FWHM linewidth of $9 \mathrm{kHz}$; the right inset shows the frequency stability of the beat note, measuring an Allan deviation of $1.15 \times 10^{-8} \cdot \sqrt{\tau}$ when referenced to the $\mathrm{THz}$ carrier. Its long-term stability can be further improved by a cost-effective computer-aided slow feedback control of the Turing sideband power. The feedback bandwidth is about $1 \mathrm{~Hz}$. As the microresonator is housed in a temperature-controlled enclosure, we attribute the instability of the Turing-roll repetition rate mainly to the effects associated with the fluctuation of intracavity power, which, at the same time, has major impact on the Turing sideband power. Thus, by stabilizing the Turing sideband power, the fluctuation of intracavity power is reduced, and

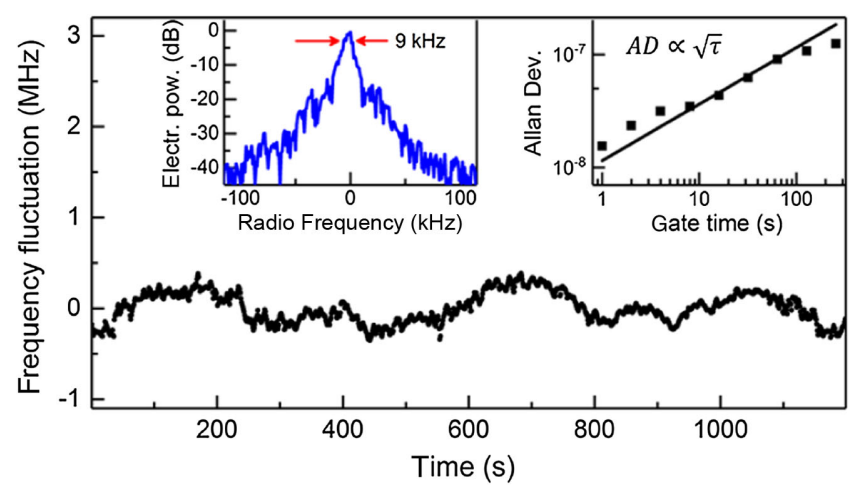

FIG. 4. Long-term frequency fluctuation of the first sideband with respect to the pump in the free-running mode, showing the repetition rate fluctuation of $160 \mathrm{kHz}$ over 20 minutes. The left inset shows the linewidth of the beat note with a sweep time of $200 \mathrm{~ms}$, measuring a narrow FWHM linewidth of $9 \mathrm{kHz}$; the right inset shows the frequency stability of the beat note, measuring an Allan deviation of $1.15 \times 10^{-8} \cdot \sqrt{\tau}$ when referenced to the $\mathrm{THz}$ carrier. 
(a)
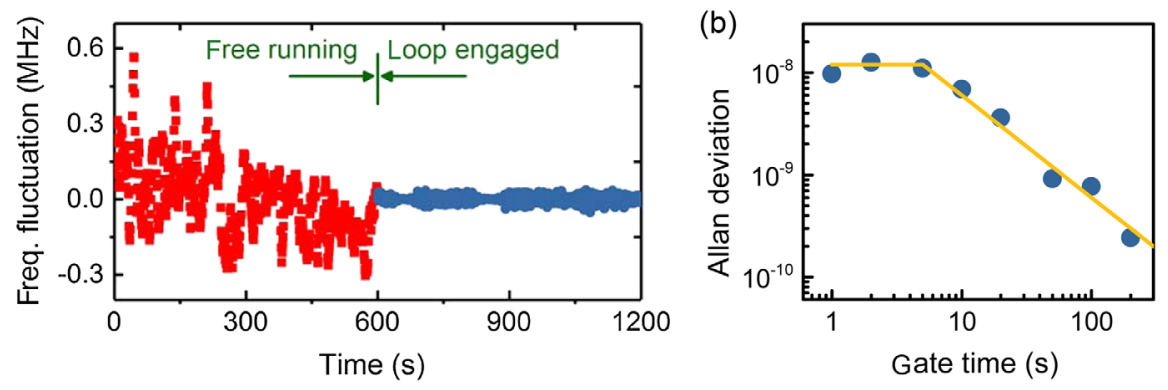

FIG. 5. (a) Long-term frequency fluctuation of the first sideband with respect to the pump engaging (blue) and without engaging (red) the computer-aided slow feedback control, counted with a gate time of 1 second. (b) Allan deviation of the stabilized THz frequency, measuring a plateau of $1.2 \times 10^{-8}$ for gate times shorter than 5 seconds and a roll-off of $6 \times 10^{-8} / \tau$ for longer gate times.

consequently, the long-term stability of the Turing-roll repetition rate is improved (Fig. S16). When the feedback loop is engaged, the frequency stability is greatly improved, as shown in Fig. 5(a). Figure 5(b) shows the Allan deviation after stabilization, measuring an improvement of more than 2 orders of magnitude and reaching $6 \times 10^{-10}$ at $100 \mathrm{~s}$ with an inverse linear dependence on the gate time.

To convert the Turing pattern into the $\mathrm{THz}$ radiation, we fabricate an ErAs:InGaAs plasmonic photomixer [Fig. 6(a)], which features a good spectral response from 0.8 to $1.6 \mathrm{THz}$ due to the logarithmic spiral antenna design [43]. A C/L WDM filter, followed by an erbium-doped fiber amplifier (EDFA), is used to selectively amplify the
Turing pattern sidebands in the 1530-1565-nm C-band range. Note that the EDFA is necessary only because our current $\mathrm{Si}_{3} \mathrm{~N}_{4}$ microresonator has a strong $Q$-factor roll-off in the C-band; thus, we are limited to pumping it in the L-band. Figure 6(b) shows four examples illustrating the tunability of the Turing-roll repetition rate (1.14$1.57 \mathrm{THz}$ ), which in turn determines the $\mathrm{THz}$ frequency, by tuning the chip temperature or the pump wavelength. Figure 6(c) plots the room-temperature radiated $\mathrm{THz}$ power as a function of the optical pump power, showing a nearly quadratic dependence even at the maximum available pump power of $54 \mathrm{~mW}$, without much saturation roll-off in the $\mathrm{THz}$ generation. Up to $600 \mu \mathrm{W} \mathrm{THz}$

(a)
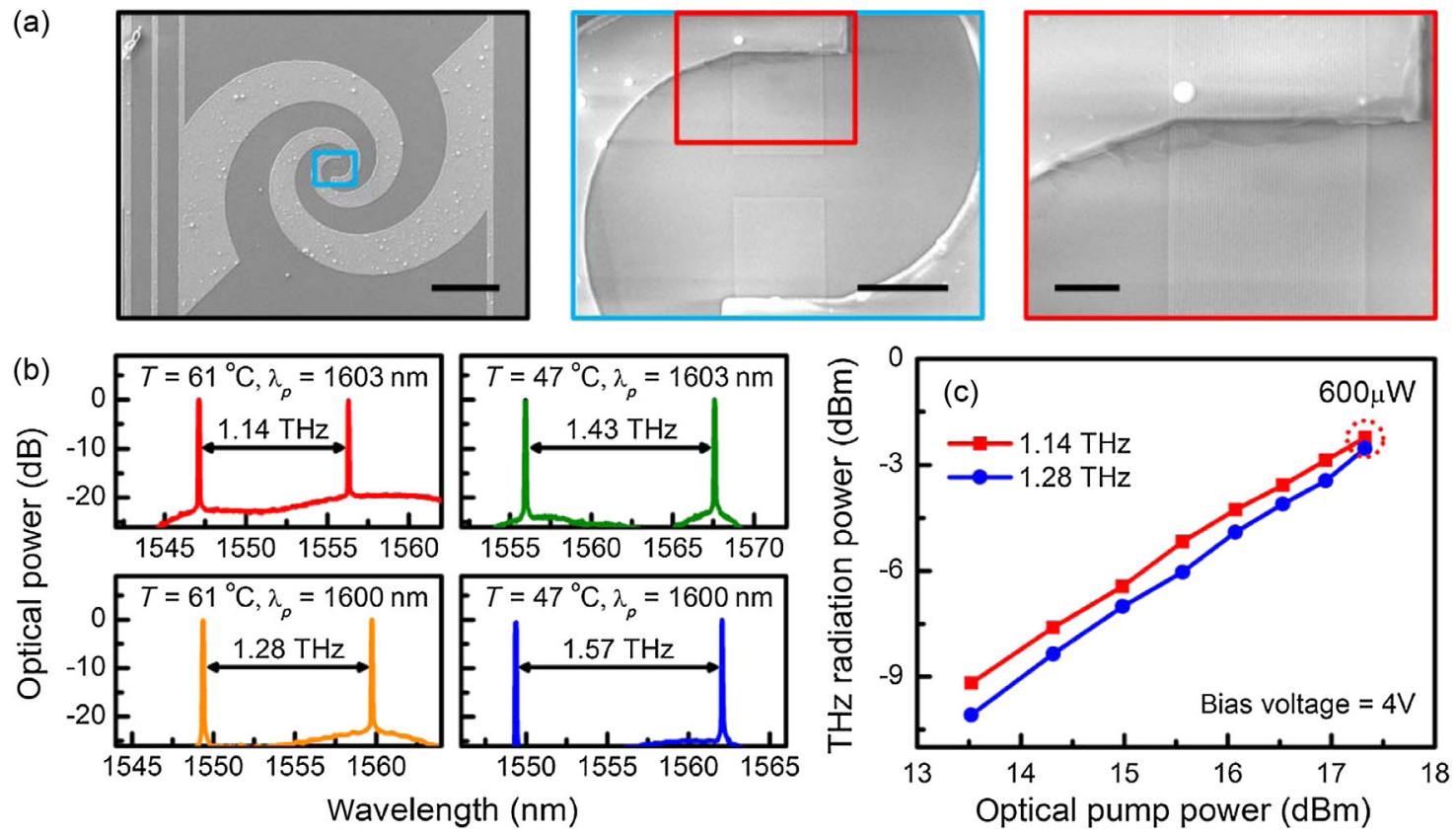

FIG. 6. (a) Scanning electron micrographs of the fabricated plasmonic photomixer with a logarithmic spiral antenna integrated with plasmonic contact electrodes on an ErAs:InGaAs substrate. Scale bars from left to right: $100 \mu \mathrm{m}, 10 \mu \mathrm{m}$, and $3 \mu \mathrm{m}$. (b) Turing-roll repetition rate, and hence the generated $\mathrm{THz}$ frequency, can be tuned by changing the pump wavelength and the resonator temperature. (c) THz radiation power as a function of optical pump power. Power-conversion efficiency of $1.1 \%$ can be obtained with an optical pump power of $54 \mathrm{~mW}$. 
radiation is generated with an optical-to- $\mathrm{THz}$ powerconversion efficiency of $1.1 \%$.

In summary, we demonstrate the scheme of incorporating the mode hybridization effect to attain globally stable microresonator Turing patterns in chip-scale nonlinear nitride cavities with significantly enlarged parameter spaces, achieving an unprecedented pump depletion and a recordhigh power-conversion efficiency of $45 \%$ with an elevated peak-to-valley contrast of 100. Controllable asymmetry in the Turing-roll spectrum is also demonstrated. The fractional frequency sideband nonuniformity of the Turing pattern is measured down to $7.3 \times 10^{-14}$, with a short-term $(200 \mathrm{~ms}$ sweep time) linewidth of $9 \mathrm{kHz}$ and a long-term (over 20 minutes) fluctuation of $160 \mathrm{kHz}$ in the free-running mode. The long-term stability can be further improved to sub-kHz with a simple computer-aided slow feedback control. We observe that the temporal shapes of the Turing patterns change with respect to pump detuning, not because of the coherence loss but because of the pump phase slip near the cavity resonance. The robustness, tunability, good coherence, and high efficiency of the demonstrated Turing pattern make it an excellent photomixer pump for narrow-linewidth-tunable $\mathrm{THz}$ radiation. Pumping a novel ErAs:InGaAs plasmonic photomixer, we then transfer the Turing pattern optical coherence to the $\mathrm{THz}$ carrier and generate up to $600 \mu \mathrm{W} \mathrm{THz}$ radiation power at room temperature. The carrier frequency is discretely tunable over a broadband from $1.14 \mathrm{THz}$ to $1.57 \mathrm{THz}$. The demonstrated coherent high-power Turing$\mathrm{THz}$ system has the potential to be the room-temperature onchip $\mathrm{THz}$ local oscillator for astrophysics, medical imaging, and wireless communication.

\section{ACKNOWLEDGMENTS}

The authors thank Bart H. McGuyer for discussions. The authors acknowledge funding support from Office of Naval Research (ONR) Grant No. N00014-14-1-0041, Air Force Office of Scientific Research (AFOSR) Young Investigator Grant No. FA9550-15-1-0081, Defense Advanced Research Projects Agency (DARPA) Grant No. HR001115-2-0014, and National Science Foundation (NSF) Grant No. PHY-1349725.

S.-W. H. and J. Y. contributed equally to this work.

\section{APPENDIX A: $\mathrm{Si}_{3} \mathrm{~N}_{4}$ MICRORESONATOR FABRICATION}

First, a $3 \mu \mathrm{m}$-thick oxide layer is deposited via plasmaenhanced chemical vapor deposition (PECVD) on $p$-type 8 " silicon wafers to serve as the under-cladding oxide. Then, low-pressure chemical vapor deposition (LPCVD) is used to deposit a 725-nm silicon nitride for the ring resonators, with a gas mixture of $\mathrm{SiH}_{2} \mathrm{Cl}_{2}$ and $\mathrm{NH}_{3}$. The resulting silicon nitride layer is patterned by optimized 248-nm deep-ultraviolet lithography and etched down to the buried oxide layer via optimized reactive-ion dry etching. Under a scanning electron microscope, the etched sidewall verticality is measured to be $88^{\circ}$, and the residual sidewall imperfections result in the mode hybridization effect described in the main text. Next, the silicon nitride ring resonators are over-cladded with a $3 \mu \mathrm{m}$-thick oxide layer, deposited initially with LPCVD $(500 \mathrm{~nm})$ and then with PECVD $(2500 \mathrm{~nm})$. The propagation loss of the silicon nitride waveguide is measured to be $0.2 \mathrm{~dB} / \mathrm{cm}$ at the pump wavelength. Cold cavity properties of the microresonator are precisely characterized by a hydrogen-cyanide-referenced swept-wavelength interferometer (Ref. [19]).

\section{APPENDIX B: ULTRAFAST INTENSITY AUTOCORRELATION AND SELF-HETERODYNE CHARACTERIZATION}

The optical intensity autocorrelator consists of a 1-mmthick $\beta$-BBO crystal, supporting a bandwidth of $200 \mathrm{~nm}$, and a silicon avalanche photodiode, supporting a sensitivity of $100 \mu \mathrm{W}$. The setup is configured in a noncollinear geometry, and careful checks are done before measurements to ensure only background-free second-harmonic signals are collected. The use of dispersive optics is also minimized such that the additional dispersion introduced to the pulse is only $-50 \mathrm{fs}^{2}$. To further confirm the coherence of the Turing sidebands, self-heterodyne linewidth measurements are performed with detailed setup, as shown in Ref. [19]. With a 200-MHz acousto-optic modulator and $24.5 \mu$ s delay provided by a $5-\mathrm{km}$ single-mode optical fiber, our self-heterodyne setup shows a minimum linewidth at $13 \mathrm{kHz}$, about 40 times smaller than the pump laser linewidth $(\approx 500 \mathrm{kHz})$.

\section{APPENDIX C: TURING PATTERN UNIFORMITY MEASUREMENTS AGAINST A FIBER FREQUENCY COMB}

Sideband frequency uniformity of the Turing pattern is measured by heterodyne referencing against a benchmark optical-fiber-laser frequency comb, Menlo FC1500-250WG. As detailed in Ref. [19], the spontaneous Turing roll is split into three beat-detection units ( $m= \pm 1$ and pump) with more than $40-\mathrm{dB}$ signal-to-noise ratio at $100-\mathrm{kHz}$ resolution bandwidth. The sideband beat notes $\left(\delta_{1}\right.$ and $\left.\delta_{2}\right)$ are mixed with the pump beat note $\left(\delta_{0}\right)$ to cancel the residual pump frequency instability, with the frequency counter operating in the ratio-counting mode to circumvent synchronization of the simultaneous beat-note measurements.

\section{APPENDIX D: PLASMONIC PHOTOMIXER FABRICATION}

The plasmonic contact electrode gratings are designed to have $200-\mathrm{nm}$ pitch, $100-\mathrm{nm}$ metal width, $5 / 45 \mathrm{~nm} \mathrm{Ti} / \mathrm{Au}$ height, and 250-nm-thick silicon nitride antireflection (AR) coating. They are patterned with electron-beam lithography 
followed by deposition of $\mathrm{Ti} / \mathrm{Au}$ and liftoff. A 250-nm silicon nitride AR coating is then deposited with PECVD. Contact vias are patterned with optical lithography and etched via dry plasma etching. Finally, the logarithmic spiral antennas and bias lines are patterned again with optical lithography, followed by deposition of $\mathrm{Ti} / \mathrm{Au}$ and liftoff. The fabricated plasmonic photomixers are then mounted on a hyper-hemispherical silicon lens to improve the $\mathrm{THz}$ radiation collection.

[1] A. M. Turing, The Chemical Basis of Morphogenesis, Phil. Trans. R. Soc. B 237, 37 (1952).

[2] S. Kondo and T. Miura, Reaction-Diffusion Model as a Framework for Understanding Biological Pattern Formation, Science 329, 1616 (2010).

[3] A. W. Liehr, Dissipative Solitons in Reaction Diffusion Systems (Springer, Berlin, Heidelberg, 2013).

[4] L. A. Lugiato and R. Lefever, Spatial Dissipative Structures in Passive Optical Systems, Phys. Rev. Lett. 58, 2209 (1987).

[5] A. J. Scroggie, W. J. Firth, G. S. McDonald, M. Tlidi, R. Lefever, and L. A. Lugiato, Pattern Formation in a Passive Kerr Cavity, Chaos Solitons Fractals 4, 1323 (1994).

[6] C. Godey, I. V. Balakireva, A. Coillet, and Y. K. Chembo, Stability Analysis of the Spatiotemporal Lugiato-Lefever Model for Kerr Optical Frequency Combs in the Anomalous and Normal Dispersion Regimes, Phys. Rev. A 89, 063814 (2014).

[7] A. Coillet, I. Balakireva, R. Henriet, K. Saleh, L. Larger, J. M. Dudley, C. R. Menyuk, and Y. K. Chembo, Azimuthal Turing Patterns, Bright and Dark Cavity Solitons in Kerr Combs Generated with Whispering-Gallery-Mode Resonators, IEEE Photonics J. 5, 6100409 (2013).

[8] A. B. Matsko, W. Liang, A. A. Savchenkov, and L. Maleki, Chaotic Dynamics of Frequency Combs Generated with Continuously Pumped Nonlinear Microresonators, Opt. Lett. 38, 525 (2013).

[9] A. Coillet and Y. K. Chembo, Routes to Spatiotemporal Chaos in Kerr Optical Frequency Combs, Chaos 24, 013113 (2014).

[10] T. Herr, V. Brasch, J. D. Jost, C. Y. Wang, N. M. Kondratiev, M. L. Gorodetsky, and T. J. Kippenberg, Temporal Solitons in Optical Microresonators, Nat. Photonics 8, 145 (2014).

[11] S.-W. Huang, H. Zhou, J. Yang, J. F. McMillan, A. Matsko, M. Yu, D.-L. Kwong, L. Maleki, and C. W. Wong, ModeLocked Ultrashort Pulse Generation from On-Chip Normal Dispersion Microresonators, Phys. Rev. Lett. 114, 053901 (2015).

[12] X. Xue, Y. Xuan, Y. Liu, P.-H. Wang, S. Chen, J. Wang, D. E. Leaird, M. Qi, and A. M. Weiner, Mode-Locked Dark Pulse Kerr Combs in Normal-Dispersion Microresonators, Nat. Photonics 9, 594 (2015).

[13] X. Yi, Q.-F. Yang, K. Y. Yang, M.-G. Suh, and K. Vahala, Soliton Frequency Comb at Microwave Rates in a High- $Q$ Silica Microresonator, Optica 2, 1078 (2015).

[14] C. Joshi, J. K. Jang, K. Luke, X. Ji, S. A. Miller, A. Klenner, Y. Okawachi, M. Lipson, and A. L. Gaeta, Thermally
Controlled Comb Generation and Soliton Modelocking in Microresonators, Opt. Lett. 41, 2565 (2016).

[15] T. J. Kippenberg, R. Holzwarth, and S. A. Diddams, Microresonator-Based Optical Frequency Combs, Science 332, 555 (2011).

[16] S.-W. Huang, J. Yang, M. Yu, B. H. McGuyer, D.-L. Kwong, T. Zelevinsky, and C. W. Wong, A Broadband Chip-Scale Optical Frequency Synthesizer at $2.7 \times 10^{-16}$ Relative Uncertainty, Sci. Adv. 2, e1501489 (2016).

[17] P. Del'Haye, A. Coillet, T. Fortier, K. Beha, D. C. Cole, K. Y. Yang, H. Lee, K. J. Vahala, S. B. Papp, and S. A. Diddams, Phase-Coherent Microwave-to-Optical Link with a Self-Referenced Microcomb, Nat. Photonics 10, 516 (2016).

[18] J. Lim, S.-W. Huang, A. K. Vinod, P. Mortazavian, M. Yu, D.-L. Kwong, A. A. Savchenkov, A. B. Matsko, L. Maleki, and C. W. Wong, A Stabilized Chip-Scale Kerr Frequency Comb via a High-Q Reference Photonic Microresonator, Opt. Lett. 41, 3706 (2016).

[19] See Supplemental Material at http://link.aps.org/ supplemental/10.1103/PhysRevX.7.041002 for measurement details and numerical analyses.

[20] J. Pfeifle, A. Coillet, R. Henriet, K. Saleh, P. Schindler, C. Weimann, W. Freude, I. V. Balakireva, L. Larger, C. Koos, and Y. K. Chembo, Optimally Coherent Kerr Combs Generated with Crystalline Whispering Gallery Mode Resonators for Ultrahigh Capacity Fiber Communications, Phys. Rev. Lett. 114, 093902 (2015).

[21] A. A. Savchenkov, A. B. Matsko, W. Liang, V. S. Ilchenko, D. Seidel, and L. Maleki, Kerr Frequency Comb Generation in Overmoded Resonators, Opt. Express 20, 27290 (2012).

[22] I. S. Grudinin, L. Baumgartel, and N. Yu, Impact of Cavity Spectrum on Span in Microresonator Frequency Combs, Opt. Express 21, 26929 (2013).

[23] Y. Liu, Y. Xuan, X. Xue, P.-H. Wang, S. Chen, A. J. Metcalf, J. Wang, D. E. Leaird, M. Qi, and A. M. Weiner, Investigation of Mode Coupling in Normal-Dispersion Silicon Nitride Microresonators for Kerr Frequency Comb Generation, Optica 1, 137 (2014).

[24] S. A. Miller, Y. Okawachi, S. Ramelow, K. Luke, A. Dutt, A. Farsi, A. L. Gaeta, and M. Lipson, Tunable Frequency Combs Based on Dual Microring Resonators, Opt. Express 23, 21527 (2015).

[25] X. Xue, Y. Xuan, P.-H. Wang, Y. Liu, D. E. Leaird, M. Qi, and A. M. Weiner, Normal-Dispersion Microcombs Enabled by Controllable Mode Interactions, Laser Photonics Rev. 9, L23 (2015).

[26] S.-W. Huang, J. Yang, J. Lim, H. Zhou, M. Yu, D.-L. Kwong, and C. W. Wong, A Low-Phase-Noise $18 \mathrm{GHz}$ Kerr Frequency Microcomb Phase-Locked over $65 \mathrm{THz}$, Sci. Rep. 5, 13355 (2015).

[27] S.-W. Huang, H. Liu, J. Yang, M. Yu, D.-L. Kwong, and C. W. Wong, Smooth Coherent Kerr Frequency Combs Generation with Broadly Tunable Pump by Higher Order Mode Suppression, Sci. Rep. 6, 26255 (2016).

[28] K. Unterrainer, A. Benz, J. Darmo, C. Deutsch, G. Fasching, J. Kroll, D. P. Kelly, M. Martl, T. Müller, W. Parz et al., Terahertz Quantum Cascade Devices: From Intersubband Transition to Microcavity Laser, IEEE J. Sel. Top. Quantum Electron. 14, 307 (2008). 
[29] S. Jung, A. Jiang, Y. Jiang, K. Vijayraghavan, X. Wang, M. Troccoli, and M. A. Belkin, Broadly Tunable Monolithic Room-Temperature Terahertz Quantum Cascade Laser Sources, Nat. Commun. 5, 4267 (2014).

[30] C. Sirtori, S. Barbieri, and R. Colombelli, Wave Engineering with THz Quantum Cascade Lasers, Nat. Photonics 7, 691 (2013).

[31] J. Darmo, V. Tamosiunas, G. Fasching, J. Kröll, K. Unterrainer, M. Beck, M. Giovannini, J. Faist, C. Kremser, and P. Debbage, Imaging with a Terahertz Quantum Cascade Laser, Opt. Express 12, 1879 (2004).

[32] S. Koenig, D. Lopez-Diaz, J. Antes, F. Boes, R. Henneberger, A. Leuther, A. Tessmann, R. Schmogrow, D. Hillerkuss, R. Palmer et al., Wireless Sub-THz Communication System with High Data Rate, Nat. Photonics 7, 977 (2013).

[33] Y. Yoshimizu, S. Hisatake, S. Kuwano, J. Terada, N. Yoshimoto, and T. Nagatsuma, Wireless Transmission Using Coherent Terahertz Wave with Phase Stabilization, IEICE Electron. Express 10, 578 (2013).

[34] A. Rueda, F. Sedlmeir, M. C. Collodo, U. Vogl, B. Stiller, G. Schunk, D. V. Strekalov, C. Marquardt, J. M. Fink, O. Painter, G. Leuchs, and H. G. L. Schwefel, Efficient Microwave to Optical Photon Conversion: An Electro-Optical Realization, Optica 3, 597 (2016).

[35] T. Nagatsuma, G. Ducournau, and C. C. Renaud, Advances in Terahertz Communications Accelerated by Photonics, Nat. Photonics 10, 371 (2016).

[36] Q. Quraishi, M. Griebel, T. Kleine-Ostmann, and R. Bratschitsch, Generation of Phase-Locked and Tunable Continuous-Wave Radiation in the Terahertz Regime, Opt. Lett. 30, 3231 (2005).

[37] J.-F. Cliche, B. Shillue, M. Tetu, and M. Poulin, A 100GHz-Tunable Photonic Millimeter Wave Synthesizer for the Atacama Large Millimeter Array Radio Telescope, in Proceedings of IEEE/MTT-S International Microwave Symposium, Honolulu, 2007 (IEEE, New York, 2007), p. 349.

[38] H.-J. Song, N. Shimizu, and T. Nagatsuma, Generation of Two-Mode Optical Signals with Broadband Frequency Tunability and Low Spurious Signal Level, Opt. Express 15, 14901 (2007).

[39] A. Rolland, G. Loas, M. Brunel, L. Frein, M. Vallet, and M. Alouini, Non-linear Optoelectronic Phase-Locked Loop for Stabilization of Opto-Millimeter Waves: Towards a Narrow Linewidth Tunable THz Source, Opt. Express 19, 17944 (2011).

[40] G. Pillet, L. Morvan, M. Brunel, F. Bretenaker, D. Dolfi, M. Vallet, J. P. Huignard, and A. L. Floch, Dual-Frequency Laser at $1.5 \mu \mathrm{m}$ for Optical Distribution and Generation of High-Purity Microwave Signals, J. Lightwave Technol. 26, 2764 (2008).

[41] G. Danion, C. Hamel, L. Frein, F. Bondu, G. Loas, and M. Alouini, Dual Frequency Laser with Two Continuously and Widely Tunable Frequencies for Optical Referencing of $\mathrm{GHz}$ to $\mathrm{THz}$ Beatnotes, Opt. Express 22, 17673 (2014).

[42] H. Shao, S. Keyvaninia, M. Vanwolleghem, G. Ducournau, X. Jiang, G. Morthier, J.-F. Lampin, and G. Roelkens, Heterogeneously Integrated III-V/Silicon Dual-Mode Distributed Feedback Laser Array for Terahertz Generation, Opt. Lett. 39, 6403 (2014).

[43] C. W. Berry, M. R. Hashemi, S. Preu, H. Lu, A. C. Gossard, and M. Jarrahi, High Power Terahertz Generation Using 1550 nm Plasmonic Photomixers, Appl. Phys. Lett. 105, 011121 (2014). 